\title{
TECHNIQUES DE BIOLOGIE MOLÉCULAIRE COMME OUTILS DE GESTION DES PÊCHES : BILAN ET PERSPECTIVES.
}

\author{
L. BERNATCHEZ \\ Institut National de la Recherche Scientifique (INRS-Eau), \\ 2800 rue Einstein, suite 105, C.P. 7500, Sainte-Foy (Québec), CANADA G1V 4C7.
}

\section{RÉSUMÉ}

Le concept de stock stipule que la diversité génétique et la structure populationnelle des espèces doivent être reconnues et préservées afin d'assurer une utilisation optimale de la ressource. Néanmoins, les programmes de gestion ont en général manqué d'objectifs clairs concernant la conservation de la diversité génétique. De plus, les chercheurs en biologie des pêches ont longtemps hésité à intégrer l'usage d'outils moléculaires dans l'étude des populations. Le résultat de cette façon de voir et de faire a mené à un retard dans le développement de l'approche génétique dans notre discipline, comparativement aux domaines médical et agricole. Dans le contexte du "Colloque franco-québécois sur l'intégration des technologies modernes à la gestion des poissons dulcicoles et amphihalins", l'objectif de cet article est d'énoncer les bénéfices possibles de l'application de techniques de biologie moléculaire en gestion des pêches. Les techniques déjà disponibles sont décrites brièvement et leur utilité est appuyée par des exemples concrets d'application. Les nouvelles approches en développement, de même que leurs domaines d'application potentielle, sont décrits.

\section{MOLECULAR BIOLOGY TECHNIQUES IN FISHERY MANAGEMENT : APPLICATIONS AND PERSPECTIVES.}

\section{SUMMARY}

The stock concept implies that genetic diversity and population structure must be considered and preserved in order to optimize the resource use. Nevertheless, management programs have generally lacked clear objectives regarding the conservation of gene pools. Furthermore, fishery biologists have often hesitated to integrate the use of molecular tools to population studies. These factors led to a slow development of a genetic approach in fisheries science when compared to other fields, such as medecine and agriculture. In the context of the "Colloque franco-québécois sur f'intégration des technologies modernes à la gestion des poissons dulcicoles et amphihalins", the main objective of this paper is to state the potential benefits of integrating molecular tools to fishery management. The already available techniques are briefly described and their usefulness supported by "real life" applications. New approaches and their potential applications are also discussed.

\section{INTRODUCTION}

Depuis la naissance du concept biologique de l'espèce, il est reconnu que les espèces sont constituées d'ensembles de populations génétiquement distinctes, évoluant plus ou moins indépendamment et pouvant ainsi développer des adaptations locales propres. Ces notions ont été intégrées en gestion des pêches sous le vocable de concept des stocks stipulant que la diversité génétique et la structure populationnelle des espèces doivent être reconnues et préservées afin d'assurer une utilisation optimale de la ressource (revue dans MACCLEAN et EVANS, 1981). Quoique conceptuellement acceptée, l'étude génétique des populations a été négligée dans les programmes de gestion qui ont en général manqué d'objectifs clairs concernant la conservation de la diversité génétique (ALLENDORF et al., 1987). 
Quand on a porté attention à l'étude de la variation populationnelle dans les programmes de gestion, ce fut le plus souvent en utilisant des méthodes indirectes telles que l'étude de la variabilité morphologique. Quoiqu'ayant ses mérites, notamment au niveau des coûts peu élevés de sa pratique, on peut s'interroger sur la valeur des informations obtenues par cette approche, compte tenu de la grande plasticité phénotypique induite par les effets environnementaux qui caractérise les espèces de poissons en général. De plus, les chercheurs en biologie des pêches ont longtemps hésité à intégrer l'usage d'outils moléculaires dans l'étude des populations. Le résultat de cette façon de voir et de faire a mené à une inertie dans le développement de l'approche génétique dans notre discipline, comparativement aux domaines médical et agricole. Le but de cet écrit est de présenter les bénéfices possibles de l'approche génétique moléculaire pour la gestion des pêches en décrivant des exemples concrets et en présentant le potentiel offert par le développement rapide de méthodes de plus en plus puissantes, mais aussi de plus en plus accessibles, tant d'un point de vue technique que financier.

\section{MÉTHODES ET RÉSULTATS D'APPLICATION}

\subsection{L'électrophorèse des protéines}

L'introduction de l'étude de la variation protéique par électrophorèse dans les années soixante a procuré la première base véritable à l'étude génétique des populations. Cette approche a été utilisée avec succès pour l'étude de la variation génétique de populations naturelles et domestiques de poissons et a généré une littérature abondante (revue dans UTTER, 1991). A titre d'exemple, l'étude intensive de la variation protéique au sein des populations sauvages et domestiques de la Truite commune (Salmo trutta $L$.) en France s'est avérée particulièrement fructueuse et a permis l'élaboration d'un plan de gestion génétique détaillé pour cette espèce (GUYOMARD, 1989a). Plus récemment, les marqueurs enzymatiques ont été utilisés avec succès en France pour décrire des mécanismes d'hybridation chez les Cyprinidés (BERREBI et CATTANEO-BERREBI, 1993), de même que pour documenter les phénomènes d'introgression entre truites ( $S$. trutta) sauvages et de repeuplement (BEAUDOU et al., 1992). Surtout parce que ses coûts sont modérés tout en permettant l'analyse rapide d'un grand nombre d'individus, l'électrophorèse protéique constitue une technique de choix. Cependant, son utilité est limitée pour l'étude de plusieurs espèces qui démontrent une diversité génétique insuffisante pour permettre des études performantes de discrimination de populations. C'est le cas par exemple pour l'Omble chevalier (Salvelinus alpinus ; MAGNUSSON et FERGUSON, 1987) ou le Bar rayé (Morone saxatilis ; SIDELL et al., 1980). Le manque de variation observée par électrophorèse protéique peut être relié à l'histoire démographique des espèces, mais également au fait que la variation observée au niveau protéique est éloignée de deux pas (traduction ADN-ARN, translation ARN-protéines) du génome, ne pouvant ainsi révéler qu'une fraction des différences s'y retrouvant.

\subsection{Les marqueurs ADN}

Le développement accéléré des techniques de biologie moléculaire depuis le début des années quatre-vingts a ouvert la voie à l'identification d'un nombre quasi illimité de marqueurs génétiques en permettant l'étude de la variation au niveau de l'ADN même. On peut distinguer trois types généraux de marqueurs $A D N$ : $A D N$ mitochondrial ( $A D N m t), A D N$ nucléaire simple copie, et $A D N$ nucléaire satellite (ADN répétitifs) qui peuvent être révélés par différentes méthodes (revue dans HALLERMAN et BECKMANN, 1988).

\subsubsection{ADN mitochondrial étudié par le polymorphisme de longueurs des fragments de restriction (RFLP)}

De toutes les nouvelles approches moléculaires, c'est celle qui a été la plus préconisée au cours de la dernière décennie même si elle demeure sous-exploitée relativement aux problèmes de gestion. Ainsi, la majorité des études effectuées à ce jour ont été préliminaires, c'est-à-dire souvent limitées dans leur ampleur, tant par la couverture du nombre d'individus analysés que par le niveau de résolution adopté.

Plusieurs caractéristiques de l'ADN mitochondrial en font un marqueur de choix pour l'étude populationnelle (revue dans MORITZ et al., 1987). C'est un petit génome de structure 
simple, présent en plusieurs copies identiques dans chaque cellule (1000-1000 000 copies) et donc, facile à isoler. Son taux de mutation nucléotidique est de 5 à 10 fois supérieur à celui du génome nucléaire, ce qui a priori augmente sa capacité de résolution. Parce qu'il est haploïde (un seul type par individu) et transmis maternellement, sa taille de population effective à l'équilibre est théoriquement $1 / 4$ de celui des gènes nucléaires et conséquemment, l'ADNmt est moins sensible à l'effet homogénéisateur du flux génique entre populations, ou inversement, plus sensible à la dérive génique, ce qui accentue son pouvoir de discrimination.

La façon la plus courante d'étudier le polymorphisme de l'ADN mitochondrial est de le faire digérer par des enzymes de restriction qui ont la capacité de reconnaître des séquences spécifiques de 4 à 8 nucléotides et de couper l'ADN là où ces sites sont rencontrés. Les fragments d'ADN résultant de cette digestion sont ensuite séparés selon leur taille par électrophorèse sur gel d'agarose ou d'acrylamide. La variation observée dans le nombre et la taille des fragments peut donc être directement interprétée comme variation génétique au niveau du génome. Ainsi, la substitution d'un nucléotide causant le gain ou la perte d'un site reconnu par une enzyme de restriction donnée sera visualisée sur le gel. De façon générale, plus on utilise d'enzymes de restriction différentes, plus on génère de caractères et plus on augmente la résolution de la technique. II existe de nombreuses façons de préparer l'ADN mitochondrial, de séparer les fragments de restriction et de les visualiser (revue dans CHAPMAN et BROWN, 1990).

Malgré son utilisation relativement restreinte, l'analyse de l'ADNmt par enzyme de restriction s'est avérée fructueuse pour l'étude populationnelle chez les poissons et ce, à différentes échelles spatiales (revue dans BILLINGTON et HEBERT, 1991). Les quelques exemples qui suivent, tirés de nos travaux, illustrent bien ces faits.

Dans une étude de la variation génétique chez le Grand Corégone (Coregonus clupeaformis), la plus importante espèce d'intérêt commercial au Canada, BERNATCHEZ et DODSON (1991) ont démontré que sur son aire de répartition nord-américaine, l'espèce est constituée d'au moins quatre groupes distincts de populations, largement allopatriques et qui ont évolué séparément pendant des dizaines, voire des centaines de milliers d'années. Il est intéressant de noter que trois de ces groupes sont présents sur le territoire québécois, ce qui en fait la région supportant la plus grande diversité génétique chez le Grand Corégone. La majorité des populations actuelles de Corégones sont issues du refuge glaciaire mississipien. Par contre, les populations du sud-ouest de la province (incluant le fleuve Saint-Laurent, le lac Champlain et le lac Saint-François dans l'Estrie) sont issues d'un refuge atlantique alors que la population naine de Corégones du Lac Témiscouata, apparentée aux populations des provinces maritimes et du nord-est des Etats-Unis, appartient à un groupe différent qui a évolué indépendamment pendant au moins les cent mille dernières années. Nos résultats ont démontré de plus que, dans certains cas, ces grands groupes sont différenciés au point où l'isolement reproducteur persiste lorsque des populations de différents groupes se retrouvent en sympatrie dans un même lac (BERNATCHEZ et DODSON, 1990a). Il apparaît donc évident qu'une gestion rationnelle devrait prendre en considération l'existence de cette diversité sous-tendant que ces groupes génétiquement distincts de populations ont pu développer des adaptations physiologiques, écologiques ou comportementales qui leur sont propres. L'existence de groupes génétiques distincts sur le territoire québécois a également été rapportée chez d'autres espèces, notamment le Touladi (Salvelinus namaycush; BILLINGTON, 1990), le Doré jaune (Stizostedion vitreum; BILLINGTON et al., 1991), et l'Éperlan arc-en-ciel (Osmerus mordax; BERNATCHEZ et al., 1994).

L'analyse de l'ADN mitochondrial s'est avérée également utile pour l'étude de discrimination des stocks à une échelle spatiale plus fine. Ainsi, dans une étude de la variation génétique au sein des stocks anadromes de Ciscos (Coregonus artedii) des baies James et Hudson exploités par les autochtones, BERNATCHEZ et DODSON (1990b) ont mis en évidence une différenciation génétique importante entre les populations peuplant les baies James et Hudson. Cette étude a aussi révélé que les rivières de la baie d'Hudson supportaient des populations distinctes alors que le flux génique entre les Ciscos capturés dans les différentes rivières de la baie James était beaucoup plus important. Ces résultats ont permis de proposer un plan de gestion dans l'éventualité d'une exploitation commerciale de ces populations.

Dans une autre étude portant sur les populations québécoises de l'Éperlan arc-en-ciel (Osmerus mordax), BERNATCHEZ (1990) et BABY et al. (1991) ont mis en évidence la différenciation génétique existant entre les populations de l'estuaire et du golfe du Saint-Laurent, supportant ainsi l'hypothèse voulant que la structure populationnelle de l'Éperlan reflète davantage 
le nombre d'aires de rétention larvaire que le nombre de sites de fraie (SINCLAIR, 1988). BABY et al. (1991) ont de plus démontré une distinction importante entre les populations d'Eperlans cantonnées en eau douce, qu'elles soient naines ou normales, et les populations anadromes. La différenciation génétique entre différents stocks a également été étudiée chez quelques autres espèces de poissons dulcicoles et amphihalins retrouvées au Québec, notamment chez l'Alose savoureuse (Alosa sapidissima; BENTZEN et al., 1989), l'Esturgeon jaune (Acipenser fulvescens ; GUÉNETTE et al., 1991) et le Poulamon atlantique (Microgadus tomcod; BABY, 1992).

\subsubsection{L'ADN mitochondrial étudié par $P C R$ et séquençage}

Quoique l'analyse de l'ADN mitochondrial par enzyme de restriction ait ouvert de nouvelles voies à l'étude génétique des populations, cette approche présente certaines limitations dans son utilisation reliée à la gestion des pêches. Notamment, elle ne permet pas de détecter le maximum de variation parce qu'un seul évènement mutationnel entraînant la perte d'un site de restriction pourra être détecté alors que plusieurs sont possibles. De plus, l'analyse par enzyme de restriction donne une valeur moyenne de la variation sur l'ensemble du génome mitochondrial et ne permet donc pas de concentrer l'analyse sur certaines portions potentiellement plus variables de la molécule. D'un point de vue pratique, ce type d'analyse requiert une quantité relativement importante de tissu prélevée par individu, préservé dans d'excellentes conditions, soit à l'état frais pour un maximum de quelques jours, ou congelé à ultra-basse température. Ceci implique en général le sacrifice des individus à analyser et certaines complications logistiques (transport d'azote liquide ou de glace carbonique) et monétaires (coût de transport) lors de campagnes d'échantillonnage sur des sites éloignés.

Une innovation technologique révolutionnaire dans le monde de la biologie moléculaire nous permet maintenant de contourner ces difficultés. La réaction de polymérase en chaîne (PCR) est un processus enzymatique simple qui permet l'amplification presqu'à volonté de segments cible d'ADN à partir d'une source infime de molécules, donc pouvant être obtenue à partir de quantités infimes (de l'ordre du $\mu \mathrm{g}$ ) de tissu (revue dans ARNHEIM et al., 1990). Brièvement, l'amplification d'ADN est réalisée en soumettant celle-ci à une série de cycles thermiques de dénaturation, d'appariement et d'élongation qui sont réalisés en présence d'une enzyme (un ADN polymérase qui effectue la synthèse de l'ADN) et en présence aussi de petits segments d'ADN (oligonucléotides) qui sont complémentaires aux régions flanquantes du segment d'ADN cible et permettent donc de le délimiter. Cette approche permet de sélectionner le segment d'ADN que l'on désire étudier et rend possible l'analyse de ce segment par séquençage direct, offrant ainsi un niveau de résolution précis au nucléotide près. Parce qu'elle ne requiert que très peu d'ADN de départ, cette méthode peut éviter le sacrifice des individus à analyser. De plus, la robustesse de cette technique élimine la nécessité de travailler avec de l'ADN de haute qualité, ce qui en permet l'obtention à partir de tissus préservés en éthanol ou autres, contournant ainsi les difficultés logistiques reliées à la conservation sur le terrain. L'analyse de l'ADN par PCR et séquençage ouvre pour la première fois la voie à l'étude génétique de spécimens de musées, populations extirpées ou espèces éteintes, préservés depuis des décennies.

Le potentiel de cette approche encore très récente demeure à peine exploré pour l'étude des populations de poissons (CARR et MARSHALL, 1991 ; MCVEIGH et al., 1991). BERNATCHEZ et al. (1992) ont utilisé la technique de PCR et de séquençage direct pour étudier la variation génétique dans un segment variable de l'ADNmt chez les populations européennes de Salmo trutta. Cette approche a permis l'obtention et l'analyse d'ADN par séquençage à partir de tissus préservés en alcool ou parfois vieux de plusieurs années. L'étude a mis en évidence l'existence de cinq groupes de truites génétiquement distincts en Europe de l'ouest, ayant évolué indépendamment pendant des milliers d'années. Au moins trois de ces groupes se retrouvent en France et sont conformes aux trois sous-unités géographiques (méditerranéenne continentale, atlantique et corse) reconnues à partir des données électrophorétiques (revue dans GUYOMARD, $1989 b$ ). Cette étude a également permis de mettre en évidence des traces de contamination génétique de populations sauvages à partir de souches domestiques.

\subsubsection{ADN nucléaire simple copie}

Comparativement à tout l'intérêt porté à l'étude de l'ADNmt au cours des dernières années, l'étude de la variabilité génétique de l'ADN nucléaire simple copie en a suscité bien peu, en 
particulier pour l'étude des populations de poissons. Les raisons de ce désintérêt sont beaucoup plus d'ordre pratique que reliées au potentiel de la méthode. Alors que les principes d'analyse par enzyme de restriction sont les mêmes pour l'ADN nucléaire que pour l'ADNmt, les techniques de visualisation des fragments de restriction de l'ADN nucléaire sont plus complexes. La digestion de l'ADN nucléaire total par enzyme de restriction génère des centaines de milliers de fragments qui sont indiscernables les uns des autres si on ne concentre pas l'analyse sur des segments restreints du génome. Ceci nécessite l'élaboration et la recherche de sondes homologues à ces segments d'ADN par techniques de clonage et la mise au point de procédés plus complexes de visualisation qui doivent être optimisés pour chaque sonde. Une fois les difficultés logistiques surmontées, il semble cependant que cette approche puisse être profitable pour l'étude populationnelle, comme dans le cas du Bar rayé (Morone saxatilis), là où d'autres approches avaient échoué (WIRGIN et MACEDA, 1991). Une des applications intéressantes de l'analyse de l'ADN nucléaire simple copie est reliée à la détermination du sexe par utilisation de sondes spécifiques aux chromosomes sexuels (FERREIRO et al., 1989). Cette approche peut entre autres permettre de contourner les difficultés de sexer les poissons avant l'apparition des caractères sexuels secondaires. Tout comme pour l'ADNmt, l'analyse de l'ADN nucléaire simple copie par amplification PCR et séquençage est envisageable quoiqu'aucun exemple d'application pour l'étude populationnelle chez les poissons n'ait été publié.

\subsubsection{ADN nucléaire satellite}

L'étude de la variation de l'ADN satellite (ADN répétitif) semble beaucoup plus prometteuse que l'analyse de l'ADN simple copie. Des découvertes récentes ont décrit, d'abord chez l'humain puis chez d'autres organismes, l'existence de loci hypervariables constitués de nombreuses petites unités de séquences répétées et constituant des régions non-codantes du génome (désigné par l'acronyme "VNTR" (variable number of tandem repeats) ; revue dans BURKE, 1989). Le taux de changement du nombre de ces séquences répétées constituant ces loci est rapide à ce point qu'il génère un très grand nombre d'allèles différents, ce qui permet l'identification génétique individuelle à un niveau de probabilité très élevé. Pour cette raison, cette technique est connue sous le nom de "DNA fingerprinting" (empreinte génétique). Outre son haut degré de résolution, cette approche offre certains autres avantages par rapport à l'analyse de l'ADN nucléaire simple copie. Entre autres, de nombreuses sondes reconnaissant ces régions hypervariables sont quasiment universelles (utiles pour de nombreuses espèces) et déjà disponibles, éliminant ainsi les processus de clonage pour la recherche de sondes. Les exemples d'application les plus spectaculaires de cette technique sont reliés à l'identification d'agresseurs à partir de traces de sang ou de sperme recueillies sur leurs victimes. Le potentiel de l'analyse de "DNA fingerprinting" pour l'étude des poissons est à peine exploré. On a entre autres utilisé cette méthode pour démontrer la perte de diversité génétique de souches domestiques de l'Achigan à grande bouche (Micropterus salmoides) relativement aux populations sauvages (WHITMORE et al., 1990). RICO et al. (1992) ont utilisé avec succès des sondes d'origine humaine et virale pour étudier les relations parentales et le succès reproducteur individuel chez l'Epinoche à trois épines (Gasterosteus aculeatus). D'autres travaux ont démontré l'utilité de telles sondes pour l'étude populationnelle chez les Salmonidés (TAGGART et FERGUSON, 1990a), Cyprinidés (GEORGES et al., 1988), Cichlidés (HARRIS et al., 1991) et Osméridés (PRODOHL et al., 1992), (TAYLOR et BENTZEN, 1993).

Un des désavantages de la technique de "DNA fingerprinting" est la révélation simultanée de la variabilité à plusieurs loci, générant ainsi des patrons de fragments d'ADN parfois complexes et difficiles d'interprétation, ce qui limite l'utilité de cette méthode pour les études populationnelles. Une façon de contourner ce problème est de développer des sondes spécifiques pour chaque locus hypervariable. Les allèles de ces loci sont ensuite révélés par hybridation avec des sondes spécifiques à chaque locus. Le développement de telles approches est cependant encore jeune et a fait l'objet de peu d'études publiées chez les poissons. Entre autres, TAGGART et FERGUSON (1990b) ont démontré chez le Saumon Atlantique (Salmo salar) que ces simples loci hypervariables comportent beaucoup plus de variation qu'aucun autre marqueur étudié à ce jour chez cette espèce.

Alternativement, les loci "VNTR" de petites tailles (microsatellites) peuvent être amplifiés spécifiquement par la technique PCR éliminant ainsi de multiples difficultés techniques reliées à l'utilisation de sondes. Cette approche, encore plus récente que la précédente, n'a fait l'objet que de deux études publiées chez les poissons. Ainsi, RICO et al. (1993) et ESTOUP et al. 
(1993) ont respectivement démontré un taux très élevé de polymorphisme de ces marqueurs chez les Épinoches et la Truite commune. Des travaux de plus grande envergure sont cependant en cours dans plusieurs laboratoires, dont le nôtre, et l'on peut déjà prévoir que l'analyse de l'ADN microsatellite sera une des approches les plus préconisées pour l'étude génétique des poissons au cours des années à venir.

\subsubsection{L'analyse des marqueurs RAPD (Random amplified polymorphic DNAs)}

Cette technique très récente permet l'analyse de l'ADN nucléaire (simple copie ou répétitif) en utilisant les propriétés de l'amplification PCR et de redondance de l'information contenue dans le code génétique (WILLIAMS et al., 1990). D'usage analogue à celui décrit pour l'analyse des empreintes satellites, l'analyse RAPD s'avère conceptuellement plus attrayante du fait :

a) qu'elle ne requiert qu'une quantité infime d'ADN source,

b) qu'aucune information préalable sur le génome sujet à l'analyse ne soit nécessaire,

c) qu'elle comporte un niveau d'expertise technique beaucoup moins complexe et, conséquemment, moins coûteux.

L'analyse RAPD n'a fait l'objet d'aucune étude publiée chez les poissons. Cependant, son utilité a été démontrée chez plusieurs groupes d'organismes (HADRYS et al., 1992) et il est à prévoir qu'elle deviendra un outil important pour l'étude des populations de poissons, notamment pour la détermination de flux géniques, de liens parentaux et d'identification de souches.

\section{DISCUSSION}

La préservation de la diversité génétique des espèces, de même que le maintien de leur structure populationnelle, sont des objectifs souhaitables à insérer en gestion des pêches et en biologie de la conservation. Il est de l'avis de l'auteur que la considération de ces objectifs dans les programmes de gestion est essentielle pour le maintien des espèces, au-delà des désirs et des intérêts souvent à court terme des utilisateurs de la ressource. Plusieurs facteurs, tels que pollution, destruction et fragmentation de l'habitat, surpêche, ou introduction d'espèces exotiques, menacent l'intégrité génétique des populations naturelles de poissons. Il a souvent fallu attendre l'extirpation totale de populations, voire même d'espèces, pour réaliser les méfaits de la perte de ressources génétiques.

L'étude de la variation génétique peut apporter beaucoup à la gestion des pêches en permettant l'identification d'entités populationnelles distinctes qui doivent être gérées séparément pour assurer la préservation à long terme et une utilisation optimale de la ressource. Ce type d'action devient particulièrement nécessaire dans le cas d'espèces faisant l'objet d'élevage pour la consommation ou le repeuplement. Par exemple, il est démontré pour Salmo trutta que la substitution des souches domestiques aux populations naturelles conduit à une perte de variabilité génétique supérieure à $50 \%$ sur les seuls bassins hydrographiques français (GUYOMARD, 1989a). La caractérisation génétique des populations peut également éclairer les gestionnaires sur l'ordre de priorité d'interventions à accorder à chacune d'elles dans un programme de conservation, selon son degré d'unicité.

Les outils moléculaires ouvrent également la voie à de nombreux autres secteurs d'application potentiellement utiles en biologie des populations. Une des voies les plus prometteuses consiste en la recherche d'association entre marqueurs génétiques ef traits phénotypiques quantitatifs. Par exemple, FERGUSON et al. (1993) ont démontré chez la Truite arc-en-ciel (Oncorhynchus mykiss) une étroite corrélation entre le génotype mitochondrial et la saison de fraie. Il est à prévoir que de plus amples recherches dans ce domaine pourront mener au développement de marqueurs génétiques associés à des propriétés de production, ce qui serait d'un grand intérêt dans les programmes de sélection ou d'ensemencement.

Un autre secteur important d'application de l'approche moléculaire est celui de l'étude de l'effet des perturbations environnementales sur l'intégrité génétique des populations. Par exemple, les effets d'hybridation et d'introgression entre espèces exotiques et populations naturelles peuvent être étudiés par les analyses d'électrophorèse enzymatique et d'ADN mitochondrial déjà disponibles. Le taux de consanguinité à l'intérieur de populations menacées ou aux tailles effectives très réduites peut également être documenté par différentes approches, notamment par celles des ADN satellites. Il est aussi possible par les techniques de PCR et séquençage d'obtenir des informations génétiques de populations extirpées à partir de spécimens 
de musées, qui peuvent être comparées à celles de populations sources potentielles pour un programme de réintroduction. L'effet des polluants sur la génétique des populations peut être documenté en étudiant la variation au niveau des oncogènes ou d'autres régions du génome en relation avec le degré de pollution de l'habitat. Eventuellement, l'étude de marqueurs associés à la croissance et la reproduction pourraient permettre de diagnostiquer si la diminution de taille induite par l'effet sélectif de la surpêche de plusieurs populations de poissons est reliée à une perte génétique irremplaçable (LAW, 1991).

\section{CONCLUSION}

La panoplie de techniques moléculaires déjà disponibles et en cours de développement rend possible l'étude de la variation génétique à tous les niveaux d'organisation, à partir des relations interspécifiques jusqu'aux différences individuelles. La pénurie d'outils disponibles ne peut donc plus être tenue pour responsable du manque de considération pour l'étude génétique des populations en gestion des pêches. La poursuite du perfectionnement de ces méthodes, et la farouche concurrence que se livrent les fournisseurs de produits et d'appareils qu'elles requièrent, allègent de plus en plus l'utilisation de ces techniques et en réduisent davantage les coûts d'application. L'analyse de l'ADN par les techniques d'amplification PCR, séquençage direct et microsatellites est un exemple marquant. Tout en offrant un niveau très élevé de résolution, ces techniques ne requièrent qu'un équipement léger, éliminant à toute fin pratique la nécessité d'acquisition d'équipements coûteux tels qu'ultracentrifugeuse ou congélateur cryogénique et réduisant considérablement les frais de campagnes d'échantillonnage. De plus en plus de chercheurs universitaires incorporent l'approche moléculaire à l'étude de la biologie des populations, formant ainsi une nouvelle génération d'étudiants "hybrides" que l'on nomme écologistes moléculaires. II serait dommage que les autorités responsables de la gestion des espèces aquatiques ne tirent pas profit de ces nouvelles ressources qui s'offrent à eux.

\section{REMERCIEMENTS}

Je tiens à remercier René GUYOMARD et Patrick BERREBI pour leurs judicieux commentaires et suggestions apportés à une version antérieure du présent manuscrit.

\section{BIBLIOGRAPHIE}

ALLENDORF F.W., RYMAN N., UTTER F., 1987. Genetic and fishery management : past, present, and future. Population Genetics and Fishery Management (Ryman N., Utter F., eds.), p. 144-160. Seattle : University of Washington Press.

ARNHEIM N., WHITE T., RAINEY W.E., 1990. Application of PCR : Organismal and population biology. BioScience, 40, 174-182.

BABY M.C., BERNATCHEZ L., DODSON J.J., 1991. Genetic structure and relationships among anadromous and landlocked populations of rainbow smelt, Osmerus mordax, Mitchill, as revealed by mtDNA restriction analysis. J. Fish. Biol., 39 (suppl. A), 61-68.

BABY M.C., 1992. Genetic variability and population structure of Atlantic tomcod, Microgadus tomcod, as revealed by mtDNA restriction analysis. Chapitre 1 , Mémoire de maîtrise, Université Laval.

BEAUDOU D., POTEAUX C., LASSERRE G., VERGNES Y., BERREBI P., 1992. Genetic impact of hatchery-reared trout (Salmo trutta fario) stocking policy in the Orb basin in the south of France. International Symposium on Genetics and Evolution of Aquatic Organisms, Bangor (U.K.), résumé.

BENTZEN P., BROWN G.C., LEGGETT W.C., 1989. Mitochondrial DNA polymorphism, population structure, and life history variation in American shad (Alosa sapidissima). Can. J. Fish. Aquat. Sci., 46, 1446-1454.

BERNATCHEZ L., 1990. Etude comparative de l'ADN mitochondrial des Eperlans arc-en-ciel (Osmerus mordax) de la région de Beaumont, de Rivière-Ouelle et de la Baie des Chaleurs durant la fraie, 1990. Rapport au Ministère du Loisir, de la Chasse et de la Pêche, Québec. $25 \mathrm{p}$. 
BERNATCHEZL., DODSON J.J., 1990a. Allopatric origin of sympatric populations of lake whitefish (Coregonus clupeaformis) revealed by mitochondrial DNA restriction analysis. Evolution, 44, 1263-1271.

BERNATCHEZ L., DODSON J.J., 1990b. Mitochondrial DNA variation among anadromous populations of Cisco (Coregonus artedii) as revealed by restriction analysis. Can. J. Fish. Aquat. Sci., 47, 533-543.

BERNATCHEZ L., DODSON J.J., 1991. Phylogeographic structure in mitochondrial DNA of the lake whitefish (Coregonus clupeaformis) and its relation to Pleistocene glaciations. Evolution, 45, 1016-1035.

BERNATCHEZ L., GUYOMARD R., BONHOMME F., 1992. DNA sequence variation of the mitochondrial control region among geographically and morphology remote European brown trout (Salmo trutta L.) populations. Mol. Ecol., 3, 161-174.

BERNATCHEZ L., MARTIN S., BERNIER A., TREMBLAY S., TRENCIA G., VERREAULT G., et VIGNEAULT Y., 1994. Conséquences de la structure génétique de l'Eperlan arc-enciel (Osmerus mordax) pour la réhabilitation de l'espèce dans l'estuaire du Saint-Laurent. Rapport technique canadien des sciences halieutiques et aquatiques. Ministère des pêches et océans, Canada, 46 p. (sous presse).

BERREBI P. et CATTANEO-BERREBI G., 1993. Natural hybridization of two species of tetraploid barbels : Barbus meridionalis and Barbus barbus (Osteichtyes, Cyprinidae) in southern France. Biol. J. Linn. Soc., 48, 319-333.

BILLINGTON N., 1990. Comparison of Lake trout (Salvelinus namaycush) from two Quebec lakes using mitochondrial DNA analysis. Rapport au Groupe Dryade, 3 p.

BILLINGTON N., HEBERT P.D.N., 1991. Mitochondrial DNA diversity in fishes and its implications for introductions. Can. J. Fish. Aquat. Sci., 48, 80-94.

BILLINGTON N., BARRETTE R.J., HEBERT P.D.N., 1992. Management implications of mitochondrial DNA variation in walleye stocks. North Am. J. Fish. Man., 12, 276-284.

BURKE T., 1989. DNA fingerprinting and other methods for the study of mating success. Trends Ecol. Evol., 7, 139-144.

CARR S.M., MARSHALL H.D., 1991 . Detection of intraspecific DNA sequence variation in the mitochondrial cytochrome $b$ gene of Atlantic cod (Gadus morhua) by the polymerase chain reaction. Can. J. Fish. Aquat. Sci., 48, 48-52.

CHAPMAN R.W., BROWN B.L., 1990. Mitochondrial isolation methods. p. 107-130. Electrophoretic and isoelectric focusing techniques in Fisheries management (Whitmore D.H., ed.). CRC Press, Boca Raton, Florida.

ESTOUP A., PRESA P., KRIEG F., VAINAM D., GUYOMARD R., 1993. (CT)n and (GT)n microsatellites : a new class of genetic markers for Salmo trutta L. (brown trout). Heredity, 71, 488-496.

FERGUSON M.M., DANZMANN R.G., ARNDT K.A., 1993. Mitochondrial DNA and allozyme variation in Ontario cultured rainbow trout spawning in different seasons. Aquaculture, 117, 237-259.

FERREIRO C., MEDRANO J.F., GALL G.A.E., 1989. Genome analysis of rainbow trout and sturgeon with restriction enzymes and hybridization with ZFY genederived probe to identify sex. Aquaculture, 81, 245-251.

GEORGES M., LEQUARRE A.S., CASTELLI M., HANSET R., VASSART G., 1988. DNA fingerprinting in domestic animals using four different minisatellite probes. Cytogenet. Cell Genet., 47, 127-131.

GUÉNETTE S., FORTIN R., 1991. Différenciation des stocks d'Esturgeon jaune (Acipenser fulvescens) du corridor fluvial du Saint-Laurent et du lac des Deux Montagnes au moyen de la morphologie et de l'ADN mitochondrial. Rapport au Ministère des Pêches et Océans et au Ministère du Loisir, de la Chasse et de la Pêche. 69 p.

GUYOMARD R., 1989a. Gestion génétique des populations naturelles : l'exemple de la Truite commune. Bull. Fr. Pêche Piscic., 314, 136-145. 
GUYOMARD R., 1989b. Diversité génétique de la Truite commune. Bull. Fr. Pêche Piscic., 314, 118-135.

HADRYS H., BALICK M., and SCHIERWATER B., 1992. Applications of random amplified polymorphic DNA (RAPD) in molecular ecology.

HALLERMAN E.M., BECKMANN J.S., 1988. DNA-level polymorphism as a tool in fisheries science. Can. J. Fish. Aquat. Sci., 45, 1075-1087.

HARRIS A.S., BIEGER S., DOYLE R.W., WRIGHT J.M., 1991. DNA fingerprinting of Tilapia, Oreochromis niloticus, and its implication to aquaculture genetics. Aquaculture, 92, 157-163.

LAW R., 1991. Fishing in evolutionary waters. New Scientist, March, 35-37.

MACCLEAN J.A., EVANS D.O., 1981. The stock concept, discreteness of fish stocks, and fisheries management. Can. J. Fish. Aquat. Sci., 38, 1889-1898.

MAGNUSSON K.P., FERGUSON M.M., 1987. Genetic analysis of four sympatric morphs of Arctic charr, Salvelinus alpinus, from Thingvallavatn, Iceland. Env. Biol. Fishes, 20, 67-73.

MCVEIGH H.P., BARTLETT S.E., DAVIDSON W.S., 1991. Polymerase chain reaction/direct sequence analysis of the cytochrome b gene in Salmo salar. Aquaculture, 95, 225-233.

MORITZ C., DOWLING T.E., BROWN W.M., 1987. Evolution of animal mitochondrial DNA: relevance for population biology and systematics. Ann. Rev. Ecol. Syst., 18, 269-292.

PRODOHL, TAGGART J.B., FERGUSON A., 1992. Genetic variability within and among sympatric brown trout (Salmo trutta) populations : multi-locus DNA analysis. Hereditas, 117, 45-50.

RICO C., KUHNLEIN U., FITZGERALD G.J., 1992. Male reproductive tactics in the Threespine stickleback : an evaluation by DNA fingerprinting. Mol. Ecol.

RICO C., ZADWORNY D., KUHNLEIN U., FITZGERALD G.J., 1993. Characterization of hypervariable microsatellite loci in the threespine stickleback Gasterosteus aculeatus. Mol. Ecol., 2, 271-272.

SIDELL B.D., OTTO R.G., POWERS D.A., KARWEIT M., SMITH J., 1980. Apparent genetic homogeneity of spawning striped bass in Upper Cheasapeake Bay. Trans. Am. Fish. Soc., 109, 99-107.

SINCLAIR M., 1988. Marine populations. An essay on population regulation and speciation. University of Washington Press, Seattle.

TAGGART J.B., FERGUSON A., 1990a. Minisatellite DNA fingerprints in salmonid fishes. Animal Gen., 21, 377-389.

TAGGART J.B., FERGUSON A., 1990b. Hypervariable minisatellite DNA single locus probes for the atlantic salmon, Salmo salar L. J. Fish. Biol., 37, 991-993.

TAYLOR E.B., and BENTZEN P., 1993. Molecular genetic evidence for reproductive isolation between sympatric populations of smelt Osmerus in Lake Utopia, south-western New Brunswick, Canada. Mol. Ecol., 2, 345-358.

UTTER F.M., 1991. Biochemical genetics and fishery management: an historical perspective. J. Fish. Biol., 39 (suppl. A), 1-20.

WHITMORE D.H., COTTON R., SHERIDAN K., 1990. DNA fingerprinting. p. 131-141. Electrophoretic and isoelectric focusing techniques in Fisheries management (Whitmore D.H., ed.). CRC Press, Boca Raton, Florida.

WILLIAMS J.G.K., KUBELIK A.R., LIVAK K.J., RAFALSKI J.A., TINGEY S.V., 1990. DNA polymorphisms amplified by arbitrary primers are useful as genetic markers. Nucl. Acids Res., 18, 6531-6535.

WIRGIN I.I., MACEDA L., 1991. Development and use of striped bass-specific RFLP probes. J. Fish. Biol., 39 (suppl. A), 159-168. 